\title{
A LOCAL STRUCTURE OF NULL HYPERSURFACES OF $\mathbb{R}_{1}^{n+1}$
}

\author{
SAMUEL SSEKAJJA*
}

\begin{abstract}
In the present paper, we characterize constant mean curvature and scalar curvature null hypersurfaces of $\mathbb{R}_{1}^{n+1}$. Locally, such hypersurfaces are a product of a null curve and generalized cylinders.
\end{abstract}

\section{INTRODUCTION}

Null geometry of submanifolds of a semi-Riemannian manifold is among the most important topics of differential geometry. This is due to their various applications in other related fields of study. For instance, null hypersurfaces appears in general relativity as models of different types of black hole horizons $[4,5,11]$. They also appear frequently in the theory of electromagnetism [4]. The study of non-degenerate submanifolds of semi-Riemannian manifolds has many similarities with the Riemannian submanifolds. However, this is not the case with null geometry. The main cause of such disparities is the degeneracy of the induced metric on the submanifold. Consequently, the study becomes more difficult and is strikingly different from the study of nondegenerate submanifolds. Some of the pioneering work on null geometry is due to Duggal-Bejancu [4], Duggal- Sahin [5] and Kupeli [13]. Such work motivated many other researchers to invest in the study of null submanifolds, for example $[1,2,3,7,8,9,10,12]$ and many more references therein.

In the books [4], [5] and [6], the authors have extensively studied the geometry of screen integrable null hypersufaces. Among such hypersurfaces are the screen conformal ones. More precisely, these are null hypersyrfaces whose local screen second fundamental forms are proportional (up to a nonvanishing function) to their local second fundamental forms. Equivalently, these are null hypersurfaces whose shape operators are proportional to those of their corresponding screen distributions. They have showed that all screen integrable null hypersurfaces of space forms are locally isometric to $\mathcal{C}_{\xi} \times M^{\prime}$, where $\mathcal{C}_{\xi}$ is a null curve and $M^{\prime}$ is a leaf

2010 Mathematics Subject Classification. Primary 53C25; Secondary 53C40, 53C50.

Key words and phrases. Null hypersurfaces, Screen conformal hypersurfaces, Isoparametric null hypersurfaces. 
of its screen distribution. In particular, it has been shown that a null cone and a Monge null hypersurfaces exhibit the above structure (see $[4,6]$ ). In the present paper, we use the ideas in [10] to extend their work by giving the details on the leaves $M^{\prime}$. To that end, we prove the following result.

Theorem 1.1. Let $(M, g)$ be an $(n+1)$-dimensional screen conformal null hypersurface of $\mathbb{R}_{1}^{n+2}: n \geq 2$, with a nonzero constant null mean curvature $\mathcal{H}_{1}$ and constant scalar curvature $r_{M}$ along its screen distribution $S(T M)$. If $\mathcal{H}_{1} \mathcal{H}_{3} \geq 0$ and $0 \leq r_{M} \leq \frac{\psi n^{2} \mathcal{H}_{1}^{2}}{2}$, then $M$ is isoparametric and locally isometric to

$$
\mathcal{C}_{\xi} \times \mathbb{S}^{1}\left(r_{1}\right) \times \mathbb{R}^{n-1} \text { or } \mathcal{C}_{\xi} \times \mathbb{S}^{2}\left(r_{2}\right) \times \mathbb{R}^{n-2},
$$

where $r_{1}^{2}=\frac{1}{2 \psi n^{2} \mathcal{H}_{1}^{2}}$ and $r_{2}^{2}=\frac{2}{\psi n^{2} \mathcal{H}_{1}^{2}}$. Here, $\mathcal{C}_{\xi}$ is a null curve tangent to the normal bundle of $M$ and $\mathcal{H}_{3}$ is the $3 r d$ order mean curvature of $M$ with respect to the screen shape operator, and $\psi$ is the screen conformality factor.

The rest of the paper is arranged as follows; In Section 2, we give the basic notions null geometry needed for this paper and in Section 3 we prove the main Theorem 1.1.

\section{PRELiminaries}

Let $(\bar{M}, \bar{g})$ be a $(n+2)$-dimensional Lorentzian manifold with index $q \in$ $\{1, \ldots, n+1\}$ and let $M$ be a hypersurface of $\bar{M}$. Let $g$ be the induced tensor field by $\bar{g}$ on $M$. Then, $M$ is called a null hypersurface of $\bar{M}$ if $g$ is of constant rank $n$ [4]. Consider the vector bundle $T M^{\perp}$ whose fibers are defined as $T_{x} M^{\perp}=\left\{Y_{x} \in T_{x} \bar{M}: \bar{g}_{x}\left(X_{x}, Y_{x}\right)=0, \quad \forall X_{x} \in T_{x} M\right\}$, for any $x \in M$. Hence, a hypersurface $M$ of $\bar{M}$ is null if and only if $T M^{\perp}$ is a distribution of rank 1 on $M$. Let $M$ be a null hypersurface, we consider the complementary distribution $S(T M)$ to $T M^{\perp}$ in $T M$, which is called a screen distribution. It is well-known that $S(T M)$ is non-degenerate (see [4]). Thus, $T M=S(T M) \perp T M^{\perp}$.

As $S(T M)$ is non-degenerate with respect to $\bar{g}$, we have $T \bar{M}=S(T M) \perp$ $S(T M)^{\perp}$, where $S(T M)^{\perp}$ is the complementary vector bundle to $S(T M)$ in $\left.T \bar{M}\right|_{M}$. Let $(M, g)$ be a null hypersurface of $(\bar{M}, \bar{g})$. Then, there exists a unique vector bundle $\operatorname{tr}(T M)$, called the null transversal bundle [4] of $M$ with respect to $S(T M)$, of rank 1 over $M$ such that for any non-zero section $E$ of $T M^{\perp}$ on a coordinate neighborhood $\mathcal{U} \subset M$, there exists a unique section $N$ of $\operatorname{tr}(T M)$ on $\mathcal{U}$ satisfying $\bar{g}(E, N)=1, \bar{g}(N, N)=\bar{g}(N, Z)=0$, for any section $Z$ of $S(T M)$. Consequently, we have the following decomposition of $T \bar{M}$.

$$
\left.T \bar{M}\right|_{M}=S(T M) \perp\left\{T M^{\perp} \oplus \operatorname{tr}(T M)\right\}=T M \oplus \operatorname{tr}(T M) .
$$


Throughout this paper, $\Gamma(E)$ will denote the $\mathcal{F}(M)$-module of differentiable sections of a vector bundle $E$. Let $\nabla$ and $\nabla^{*}$ denote the induced connections on $M$ and $S(T M)$, respectively, and $P$ be the projection of $T M$ onto $S(T M)$, then the local Gauss-Weingarten equations of $M$ and $S(T M)$ are the following [4].

$$
\begin{aligned}
& \bar{\nabla}_{X} Y=\nabla_{X} Y+h(X, Y)=\nabla_{X} Y+B(X, Y) N, \\
& \bar{\nabla}_{X} N=-A_{N} X+\nabla_{X}^{t} N=-A_{N} X+\tau(X) N, \\
& \nabla_{X} P Y=\nabla_{X}^{*} P Y+h^{*}(X, P Y)=\nabla_{X}^{*} P Y+C(X, P Y) \xi, \\
& \nabla_{X} \xi=-A_{\xi}^{*} X+\nabla_{X}^{* t} \xi=-A_{\xi}^{*} X-\tau(X) \xi, \quad A_{\xi}^{*} \xi=0,
\end{aligned}
$$

for all $X, Y \in \Gamma(T M), \xi \in \Gamma\left(T M^{\perp}\right)$ and $N \in \Gamma(\operatorname{tr}(T M))$, where $\bar{\nabla}$ is the LeviCivita connection on $\bar{M}$. In the above setting, $B$ is the local second fundamental form of $M$ and $C$ is the local second fundamental form on $S(T M) . A_{N}$ and $A_{\xi}^{*}$ are the shape operators on $T M$ and $S(T M)$ respectively, while $\tau$ is a 1-form on $T M$. The above shape operators are related to their local fundamental forms by $g\left(A_{E}^{*} X, Y\right)=B(X, Y), \quad g\left(A_{N} X, P Y\right)=C(X, P Y)$, for any $X, Y \in \Gamma(T M)$. Moreover, $\bar{g}\left(A_{E}^{*} X, N\right)=0$, and $\bar{g}\left(A_{N} X, N\right)=0$, for all $X \in \Gamma(T M)$. From these relations, we notice that $A_{E}^{*}$ and $A_{N}$ are both screen-valued operators. Let $\vartheta=\bar{g}(N, \cdot)$ be a 1 -form metrically equivalent to $N$ defined on $\bar{M}$. Take $\eta=i^{*} \vartheta$ to be its restriction on $M$, where $i: M \rightarrow \bar{M}$ is the inclusion map. Then it is easy to show that

$$
\left(\nabla_{X} g\right)(Y, Z)=B(X, Y) \eta(Z)+B(X, Z) \eta(Y),
$$

for all $X, Y, Z \in \Gamma(T M)$. Consequently, $\nabla$ is generally not a metric connection with respect to $g$. However, the induced connection $\nabla^{*}$ on $S(T M)$ is a metric connection.

Denote by $\bar{R}, R$ and $R^{*}$ the curvature tensors of the connection $\bar{\nabla}$ on $\bar{M}$, and the induced linear connections $\nabla$ and $\nabla^{*}$ on $M$ and $S(T M)$, respectively. Using the Gauss-Weingarten formulae, we obtain the following Gauss-Codazzi equations for $M$ and $S(T M)$ (see details in [4, 5]).

$$
\begin{aligned}
\bar{g}(\bar{R}(X, Y) Z, P W) & =g(R(X, Y) Z, P W) \\
& +B(X, Z) C(Y, P W)-B(Y, Z) C(X, P W), \\
g(R(X, Y) P Z, P W) & =g\left(R^{*}(X, Y) P Z, P W\right) \\
& +C(X, P Z) B(Y, P W)-C(Y, P Z) B(X, P W),
\end{aligned}
$$

for all $X, Y, Z \in \Gamma(T M), \xi \in \Gamma\left(T M^{\perp}\right)$ and $N \in \Gamma(\operatorname{tr}(T M))$.

A null hypersurface $(M, g)$ of a semi-Riemannian manifold $(\bar{M}, \bar{g})$ is called screen conformal [4, p. 51] if there exist a non-vanishing smooth function $\psi$ on 
a neighborhood $\mathcal{U}$ in $M$ such that $A_{N}=\psi A_{E}^{*}$, or equivalently, $C(X, P Y)=$ $\psi B(X, Y)$, for all $X, Y \in \Gamma(T M)$. We say that $M$ is screen homothetic if $\psi$ is a constant function on $M$. The screen distribution on a screen conformal null hy persurface $(M, g)$, of a semi-Riemannian manifold $\bar{M}$, is integrable. Moreover, the following remark holds in general for screen integrable null hypersurfaces.

Remark 2.1 ([6]). Just as in the well-known case of locally product Riemannian or semi-Riemannian manifolds, if $S(T M)$ is integrable then $M$ is locally a product manifold $\mathcal{C}_{\xi} \times M^{\prime}$ where $\mathcal{C}_{\xi}$ is a null curve tangent to $T M^{\perp}$ and $M^{\prime}$ is a leaf of $S(T M)$.

Example 2.2. The null cone and null Monge hypersurfaces of $\mathbb{R}_{1}^{n+2}$ are screen conformal, and with the structure in Remark 2.1, see [6] for details.

Throughout this paper, we use the following range of indices;

$$
1 \leq \alpha, \beta, \gamma, \ldots \leq n, \quad 0 \leq i, j, k, \ldots \leq n .
$$

Next, suppose that $(M, g)$ is a screen conformal null hypersurface of $\mathbb{R}_{1}^{n+2}$. Let $\left\{Z_{\alpha}\right\}_{\alpha=1}^{n}$ be an orthonormal principal basis of $S(T M)$, which diagonalizes $A_{\xi}^{*}$ at $x \in M$. Suppose that $\lambda_{\alpha}: 1 \leq \alpha \leq n$ are the corresponding principal curvatures.

Then the sectional curvature $\kappa\left(Z_{\alpha}, Z_{\beta}\right)$ of a plane spanned by $Z_{\alpha}$ and $Z_{\beta}$ is obtained from (2.5) and (2.6) as;

$$
\begin{aligned}
\kappa\left(Z_{\alpha}, Z_{\beta}\right) & =B\left(Z_{\beta}, Z_{\beta}\right) C\left(Z_{\alpha}, Z_{\alpha}\right)+B\left(Z_{\alpha}, Z_{\alpha}\right) C\left(Z_{\beta}, Z_{\beta}\right) \\
& -C\left(Z_{\alpha}, Z_{\beta}\right) B\left(Z_{\beta}, Z_{\alpha}\right)-B\left(Z_{\alpha}, Z_{\beta}\right) C\left(Z_{\beta}, Z_{\alpha}\right) \\
& =2 \psi \lambda_{\alpha} \lambda_{\beta},
\end{aligned}
$$

in which we have used the fact that $\bar{R}=0$. We denote by $\left|A_{\xi}^{*}\right|^{2}$ the squared norm of $A_{\xi}^{*}$ with respect to $S(T M)$. Notice that this is also the trace of the square of $A_{\xi}^{*}$. A screen conformal null hypersurface of a semi-Riemannian manifolds of constant curvature admits a symmetric Ricci tensor [5]. By a direct calculation, using (2.5), the scalar curvature $r_{M}$ of $M$ is given by

$$
r_{M}=\psi\left(n^{2} \mathcal{H}_{1}^{2}-\left|A_{\xi}^{*}\right|_{s}^{2}\right) .
$$

The shape operator $A_{\xi}^{*}$ is symmetric on $M$, and therefore diagonalizable with $n$ real-valued principal curvatures $\lambda_{1}, \ldots, \lambda_{n}$ with respect to the principal vector fields $Z_{1}, \ldots, Z_{n}$ tangent to $S(T M)$. For $0 \leq k \leq n$, let $S_{r}$ denote the $r$-th elementary symmetric function on the principal curvatures $\lambda_{1}, \ldots, \lambda_{n}$; this way, one gets $n$ smooth functions $S_{r}: M \longrightarrow \mathbb{R}$, such that $\operatorname{det}\left(t I-A_{\xi}^{*}\right)=$ $\sum_{k=0}^{n}(-1)^{k} S_{k} t^{n-k}$, where $S_{0}=1$ by definition and $I$ is the identity on $S(T M)$. 
One immediately sees that $S_{r}=\sigma_{r}\left(\lambda_{1}, \ldots, \lambda_{n}\right)$, where $\sigma_{r}=\mathbb{R}\left[\lambda_{1}, \ldots, \lambda_{n}\right]$, is the $r$-th elementary symmetric polynomial on the indeterminates $\lambda_{1}, \ldots, \lambda_{n}$. For $1 \leq r \leq n$, one defines the $r$-th mean curvature $\mathcal{H}_{r}$ of $M$ by

$$
\left(\begin{array}{l}
n \\
r
\end{array}\right) \mathcal{H}_{r}=S_{r}=\sigma_{r}\left(\lambda_{1}, \ldots, \lambda_{n}\right) .
$$

Sometimes, $S_{r}$ instead of $\mathcal{H}_{r}$ is referred to as the $r$-th mean curvature. In that regard, we will adopt the latter for mean curvature in this paper.

Next, the $r$-th Newton transformation $T_{r}$, for $0 \leq r \leq n$, on $S(T M)$ is defined by setting $T_{0}=I$ and, for $1 \leq r \leq n$, by the recurrence relation

$$
T_{r}=S_{r} I-A_{\xi}^{*} \circ T_{r-1} .
$$

By Caylay-Hamiliton theorem $T_{n}=0$. Since $T_{r}$ is a polynomial in $A_{\xi}^{*}$ for every $r$, it is also self-adjoint and commutes with $A_{\xi}^{*}$. Therefore, the basis $\left\{Z_{\alpha}\right\}_{\alpha=1}^{n}$ diagonalizes $T_{r}$. Let $\operatorname{tr}(\cdot)$ denote the trace with respect to $S(T M)$. Then, the Newton transformation $T_{r}$ satisfy the following relations (see [?] for details);

$$
\operatorname{tr}\left(A_{\xi}^{*} \circ T_{r}\right)=(r+1) S_{r+1}, \operatorname{tr}\left(A_{E}^{* 2} \circ T_{r}\right)=S_{1} S_{r+1}-(r+2) S_{r+2} .
$$

Next, we define some differential operators on $S(T M)$. To that end, we define the gradient $\nabla^{s} f$, Hessian, $\operatorname{Hess}^{s} f$, and D' Alambertian $\Delta^{s} f$ of a smooth function $f$ on $\mathcal{U} \subset M$ with respect to the screen distribution $S(T M)$ as

$$
\begin{gathered}
\nabla^{s} f=g^{\alpha \beta}\left(Z_{\alpha} f\right) Z_{\beta}, \quad \operatorname{Hess}^{s} f=Z_{\alpha}\left(Z_{\beta}(f)\right)-\left(\nabla_{Z_{\alpha}}^{*} Z_{\beta}\right) f \\
\Delta^{s} f=\operatorname{tr}\left(\operatorname{Hess}^{s} f\right)=g^{\alpha \beta}\left(Z_{\alpha}\left(Z_{\beta} f\right)-\left(\nabla_{X_{\alpha}}^{*} X_{\beta}\right) f\right),
\end{gathered}
$$

where $\left\{Z_{\alpha}\right\}_{\alpha=1}^{n}$ is a basis of $S(T M)$ and $\operatorname{tr}(\cdot)$ denotes the trace with respect to $S(T M)$.

\section{Proof OF MAIN Result: TheOREM 1.1}

Let $\left\{Z_{\alpha}\right\}_{\alpha=1}^{n}$ be an othonormal basis of $S(T M)$ which diagonalizes $A_{\xi}^{*}$ at a point $x \in M$. Let further $\left(\nabla_{Z_{\alpha}}^{*} Z_{\beta}\right)(x)=0$ and $C\left(\xi, Z_{\alpha}\right)=0$ at $x \in M$. We also assume the 1-form $\tau$ vanishes on $S(T M)$. Set $B_{\alpha \beta}:=B\left(X_{\alpha}, Z_{\beta}\right)$, then the following proposition is fundamental to our main result.

Proposition 3.1. Let $(M, g)$ be a screen integrable null hypersurface of $\mathbb{R}_{1}^{n+2}$. Then the D' Alambertian of $\left|A_{\xi}^{*}\right|_{s}^{2}$ with respect to $S(T M)$ satisfy

$$
\Delta^{s}\left|A_{\xi}^{*}\right|_{s}^{2}=2 B_{\alpha \beta} \operatorname{Hess}^{s} S_{1}-2 a\left|A_{\xi}^{*}\right|_{s}^{2}+2\left|\nabla^{s} B\right|_{s}^{2}+2 S_{1} \operatorname{tr}\left(A_{\xi}^{* 2} \circ A_{N}\right),
$$

for all $1 \leq \alpha, \beta \leq n$, where $a:=\operatorname{tr}\left(A_{\xi}^{*} \circ A_{N}\right)$. 
Proof. By the fact $\bar{R}=0$, the Gauss-Codazzi equation (3.1) of null hypersurfaces given in [4, p. 93] implies

$$
\left(\nabla_{X} h\right)(Y, Z)=\left(\nabla_{Y} h\right)(X, Z)
$$

for all $X, Y, Z \in \Gamma(T M)$, where

$$
\left(\nabla_{X} h\right)(Y, Z)=\nabla_{X}^{t} h(Y, Z)-h\left(\nabla_{X} Y, Z\right)-h\left(Y, \nabla_{X} Z\right) .
$$

As $\nabla_{Z_{\alpha}}^{*} Z_{\beta}=0$, we have

$$
h\left(\nabla_{Z_{\alpha}} Z_{\beta}, Z_{\gamma}\right)=h\left(\nabla_{Z_{\alpha}}^{*} Z_{\beta}, Z_{\gamma}\right)+C\left(Z_{\alpha}, Z_{\beta}\right) h\left(\xi, Z_{\gamma}\right)=0
$$

in which we have considered (2.3) and the fact $h(\xi, X)=0$, for any $X \in \Gamma(T M)$. Thus, (3.1) and (3.2) implies

$$
\nabla_{Z_{\gamma}}^{t} h\left(Z_{\alpha}, Z_{\beta}\right)=\nabla_{Z_{\alpha}}^{t} h\left(Z_{\gamma}, Z_{\beta}\right)
$$

Differentiating (3.3) and applying the definition of curvature, we get

$$
\begin{aligned}
& \nabla_{Z_{\mu}}^{t} \nabla_{Z_{\gamma}}^{t} h\left(Z_{\alpha}, Z_{\beta}\right)=\nabla_{Z_{\mu}}^{t} \nabla_{Z_{\alpha}}^{t} h\left(Z_{\gamma}, Z_{\beta}\right) \\
& =\nabla_{Z_{\alpha}}^{t} \nabla_{Z_{\mu}}^{t} h\left(Z_{\gamma}, Z_{\beta}\right)-\left(R\left(Z_{\alpha}, Z_{\mu}\right) h\right)\left(Z_{\beta}, Z_{\gamma}\right)
\end{aligned}
$$

where

$$
\begin{aligned}
& \left(R\left(Z_{\alpha}, Z_{\mu}\right) h\right)\left(Z_{\beta}, Z_{\gamma}\right)=R^{t}\left(Z_{\alpha}, Z_{\mu}\right) h\left(Z_{\beta}, Z_{\gamma}\right) \\
& \quad-h\left(R\left(Z_{\alpha}, Z_{\mu}\right) X_{\beta}, Z_{\gamma}\right)-h\left(Z_{\beta}, R\left(Z_{\alpha}, Z_{\mu}\right) Z_{\gamma}\right)
\end{aligned}
$$

and $R^{t}$ is the curvature tensor of the transversal bundle, given by

$$
R^{t}(X, Y) N=\nabla_{X}^{t} \nabla_{Y}^{t} N-\nabla_{Y}^{t} \nabla_{X}^{t} N-\nabla_{[X, Y]}^{t} N
$$

for any $X, Y \in \Gamma(T M)$ and $N \in \Gamma(\operatorname{tr}(T M))$. Then applying (3.4), (3.5) and (3.6) while considering $\nabla_{X}^{t} N=\tau(X) N$, we get

$$
\begin{aligned}
\nabla_{Z_{\mu}}^{t} \nabla_{Z_{\gamma}}^{t} h\left(Z_{\alpha}, Z_{\beta}\right) & =\nabla_{Z_{\alpha}}^{t} \nabla_{Z_{\mu}}^{t} h\left(Z_{\beta}, Z_{\gamma}\right)+h\left(R\left(Z_{\alpha}, Z_{\mu}\right) Z_{\beta}, Z_{\gamma}\right) \\
& +h\left(Z_{\beta}, R\left(Z_{\alpha}, Z_{\mu}\right) Z_{\gamma}\right) \\
& =\nabla_{Z_{\alpha}}^{t} \nabla_{Z_{\beta}}^{t} h\left(Z_{\mu}, Z_{\gamma}\right)+h\left(R\left(Z_{\alpha}, Z_{\mu}\right) Z_{\beta}, Z_{\gamma}\right) \\
& +h\left(Z_{\beta}, R\left(Z_{\alpha}, Z_{\mu}\right) Z_{\gamma}\right)
\end{aligned}
$$

where in the last equality we have used (3.3). Now, using the fact $h(X, Y)=$ $B(X, Y) N, B(X, Y)=g\left(A_{\xi}^{*} X, Y\right)$, for any $X, Y \in \Gamma(T M)$, and the assumption 
$\tau=0$ on the screen distribution, (3.7) reduces to

$$
\begin{aligned}
X_{\mu}\left(X_{\gamma}\left(B_{\alpha \beta}\right)\right) & =X_{\alpha}\left(X_{\beta}\left(B_{\mu \gamma}\right)\right)+B\left(R\left(X_{\alpha}, X_{\mu}\right) X_{\beta}, X_{\gamma}\right) \\
& +B\left(X_{\beta}, R\left(X_{\alpha}, X_{\mu}\right) X_{\gamma}\right) \\
& =X_{\alpha}\left(X_{\beta}\left(B_{\mu \gamma}\right)\right)+g\left(A_{\xi}^{*} X_{\gamma}, R\left(X_{\alpha}, X_{\mu}\right) X_{\beta}\right) \\
& +g\left(A_{\xi}^{*} X_{\beta}, R\left(X_{\alpha}, X_{\mu}\right) X_{\gamma}\right) .
\end{aligned}
$$

Next, as $\bar{R}=0$, from the Gauss-Codazzi relation (2.5), we get

$$
g(R(X, Y) Z, P W)=B(Y, Z) C(X, P W)-B(X, Z) C(Y, P W),
$$

for any $X, Y, Z, W \in \Gamma(T M)$. Applying (3.9) to (3.8), reduces it to

$$
\begin{aligned}
Z_{\mu}\left(Z_{\gamma}\left(B_{\alpha \beta}\right)\right) & =Z_{\alpha}\left(Z_{\beta}\left(B_{\mu \gamma}\right)\right)+B_{\mu \beta} C\left(Z_{\alpha}, A_{\xi}^{*} Z_{\gamma}\right) \\
& -B_{\alpha \beta} C\left(Z_{\mu}, A_{\xi}^{*} Z_{\gamma}\right)+B_{\mu \gamma} C\left(Z_{\alpha}, A_{\xi}^{*} Z_{\beta}\right) \\
& -B_{\alpha \gamma} C\left(Z_{\mu}, A_{\xi}^{*} Z_{\beta}\right) .
\end{aligned}
$$

Since we are working locally around a point $p \in M$, then $\tau\left(\left[Z_{\alpha}, Z_{\beta}\right]\right)=0$. Putting this into account, the assumption $\tau\left(Z_{\alpha}\right)=0$, for all $1 \leq \alpha \leq n$, and $\bar{R}=0$, we have from the Gauss-Codazzi equation (3.12) of $[4$, p. 95] that

$$
C\left(Z_{\alpha}, A_{\xi}^{*} Z_{\gamma}\right)=C\left(Z_{\gamma}, A_{\xi}^{*} Z_{\alpha}\right)
$$

Finally, placing (3.11) in (3.10) and taking trace with respect to $\mu$ and $\gamma$, we obtain

$$
\Delta^{s} B_{\alpha \beta}=\operatorname{Hess}^{s} S_{1}-\operatorname{tr}\left(A_{\xi}^{*} \circ A_{N}\right) B_{\alpha \beta}+S C\left(Z_{\alpha}, A_{\xi}^{*} Z_{\beta}\right) .
$$

Considering $g^{\alpha \beta}(x)=\delta_{\alpha \beta}$, we have $\left|A_{\xi}^{*}\right|_{s}^{2}=g^{\alpha \mu} g^{\gamma \beta} B_{\alpha \mu} B_{\gamma \beta}=B_{\alpha \beta} B_{\alpha \beta}$. Using this relation and (3.12), we have

$$
\begin{aligned}
\Delta^{s}\left|A_{\xi}^{*}\right|_{s}^{2} & =2 B_{\alpha \beta} \Delta^{s} B_{\alpha \beta}+2\left|\nabla^{s} B\right|_{s}^{2} \\
& =2 B_{\alpha \beta} \operatorname{Hess}^{s} S_{1}-2 a\left|A_{\xi}^{*}\right|_{s}^{2}+2\left|\nabla^{s} B\right|_{s}^{2}+2 S_{1} \operatorname{tr}^{s}\left(A_{\xi}^{* 2} \circ A_{N}\right),
\end{aligned}
$$

where $a:=\operatorname{tr}\left(A_{\xi}^{*} \circ A_{N}\right)$, which completes the proof.

3.1. Proof of Theorem 1.1. Setting $r=2$ in the second relation of (3.12) and considering (2.9), we have

$$
\operatorname{tr}\left(A_{\xi}^{* 3}\right)=S_{1}\left|A_{\xi}^{*}\right|_{s}^{2}-n \mathcal{H}_{1} S_{2}+3 S_{3} .
$$

On the other hand, setting $r=0$ in the second relation of (3.12), we get $\left|A_{\xi}^{*}\right|_{s}^{2}=$ $n^{2} \mathcal{H}_{1}^{2}-2 S_{2}$. Considering this last relation in (3.13), we get

$$
2 \operatorname{tr}\left(A_{\xi}^{* 3}\right)=n \mathcal{H}_{1}\left(3\left|A_{\xi}^{*}\right|_{s}^{2}-n^{2} \mathcal{H}_{1}^{2}\right)+6 S_{3} .
$$


As $\mathcal{H}_{1}$ and $r_{M}$ are constants along $S(T M)$ and $M$ is screen homothetic, it follows from (2.8) that $\left|A_{\xi}^{*}\right|_{s}^{2}$ is constant too along $S(T M)$. By the assumptions $\mathcal{H}_{1} \mathcal{H}_{3} \geq 0$ and $0 \leq r_{M} \leq \frac{\psi n^{2} \mathcal{H}_{1}^{2}}{2}$, we deduce from Proposition 3.1 and (3.14) that

$$
\begin{aligned}
\frac{1}{2 \psi} & \Delta^{s}\left|A_{\xi}^{*}\right|_{s}^{2}=\frac{1}{\psi}\left|\nabla^{s} B\right|_{s}^{2}-\left(\left|A_{\xi}^{*}\right|_{s}^{4}-\frac{3 n^{2} \mathcal{H}_{1}^{2}}{2}\left|A_{\xi}^{*}\right|_{s}^{2}+\frac{n^{4} \mathcal{H}_{1}^{4}}{2}\right)+3 n \mathcal{H}_{1} S_{3} \\
& =\frac{1}{\psi}\left|\nabla^{s} B\right|_{s}^{2}-\left(\left|A_{\xi}^{*}\right|_{s}^{2}-n^{2} \mathcal{H}_{1}^{2}\right)\left(\left|A_{\xi}^{*}\right|_{s}^{2}-\frac{n^{2} \mathcal{H}_{1}^{2}}{2}\right)+3 n \mathcal{H}_{1} S_{3}, \\
& =\frac{1}{\psi}\left|\nabla^{s} B\right|_{s}^{2}+\frac{r_{M}}{\psi^{2}}\left(\frac{\psi n^{2} \mathcal{H}_{1}^{2}}{2}-r_{M}\right)+\frac{n^{2}(n-1)(n-2)}{2} \mathcal{H}_{1} \mathcal{H}_{3} \\
& \geq \frac{1}{\psi}\left|\nabla^{s} B\right|_{s}^{2} .
\end{aligned}
$$

Notice that the left hand side of (3.15) vanishes. Since the assumption $0 \leq r_{M} \leq$ $\frac{\psi n^{2} \mathcal{H}_{1}^{2}}{2}$ also implies that $0 \leq \frac{2 r_{M}}{n^{2} \mathcal{H}_{1}^{2}} \leq \psi$, (3.15) gives $\left|\nabla^{s} B\right|_{s}^{2}=0$. As $S(T M)$ is Riemannian, this implies that $\nabla^{s} B=0$. Consequently, $M$ is isoparametric in the language of [10]. Furthermore, $r_{M}=0$ or $r_{M}=\frac{\psi n^{2} \mathcal{H}_{1}^{2}}{2}$ and by [10], the leaves of $S(T M)$ are isometric to $\mathbb{S}^{m}(r) \times \mathbb{R}^{n-m}$, for some positive $r$. Notice, from (2.8) and (2.7), that the case $r_{M}$ corresponds to $m=1$ and $r^{2}=r_{1}^{2}=\frac{1}{2 \psi n^{2} \mathcal{H}_{1}^{2}}$ and therefore, we have the structure $\mathbb{S}^{1}\left(r_{1}\right) \times \mathbb{R}^{n-1}$ for the leaves of $S(T M)$. Using a similar argument on $r_{M}=\frac{\psi n^{2} \mathcal{H}_{1}^{2}}{2}$, we obtain the structure $\mathbb{S}^{2}\left(r_{2}\right) \times \mathbb{R}^{n-2}$, where $r^{2}=r_{2}^{2}=\frac{2}{\psi n^{2} \mathcal{H}_{1}^{2}}$. Hence, our result follows from Remark 2.1.

\section{REFERENCES}

[1] C. Atindogbé, M. M. Harouna and J. Tossa, Lightlike hypersurfaces in Lorentzian manifolds with constant screen principal curvatures. Afr. Diaspora J. Math 16 (2), (2014), 31-45.

[2] C. Atindogbé, Scalar curvature on lightlike hypersurfaces, Balkan Society of Geometers, Applied Sciences, 11(2009), 9-18.

[3] M. Hassirou, Kaehler lightlike submanifolds, Journal of Mathematical Sciences: Advances and Applications, Volume 10, Number 1/2, (2011), 1-21.

[4] K. L. Duggal and A. Bejancu, Lightlike submanifolds of semi-Riemannian manifolds and applications, Kluwer Academic Publishers, 1996.

[5] K. L. Duggal and B. Sahin, Differential geometry of lightlike submanifolds. Frontiers in Mathematics, Birkhäuser Verlag, Basel, 2010.

[6] K. L. Duggal and D. H. Jin, Null curves and hypersurfaces of semi-Riemannian manifolds, World Scientific, 2007.

[7] D. H. Jin, Ascreen lightlike hypersurfaces of an indefinite Sasakian manifold, J. Korean Soc. Math. Educ. Ser. B: Pure Appl. Math. Volume 20, 1(2013), 25-35.

[8] D. H. Jin, Geometry of lightlike hypersurfaces of an indefinite sasakian manifold, Indian J. Pure Appl. Math., 41(4) (2010), 569-581. 
[9] F. Massamba and S. Ssekajja, Quasi generalized CR-lightlike submanifolds of indefinite nearly Sasakian manifolds, Arab. J. Math. 5 (2016) 87-101.

[10] S. Ssekajja, On the isoperimetric null hypersurfaces of Lorentzian manifolds, Preprint.

[11] B. ONeill, Semi-Riemannian Geometry, with Applications to Relativity. New York: Academic Press (1983).

[12] M. Navarro, O. Palmas and D. A. Solis, Null screen isoparametric hypersurfaces in Lorentzian space forms, Mediterr. J. Math. (2018) 15:215.

[13] D. N. Kupeli, Singular semi-Riemannian geometry, Mathematics and Its Applications, Vol. 366, Kluwer Academic Publishers, 1996.

* School of Mathematics

UNIVERSITY OF THE WITWATERSRAND

Private Bag 3, Wits 2050

SOUTH AFRICA

Email address: ssekajja.samuel.buwaga@aims-senegal.org 